\title{
Adsorption of monovalent and multivalent cations and anions on DNA molecules
}

\author{
E. Allahyarov, ${ }^{1}$ H. Löwen, ${ }^{2}$ and G. Gompper ${ }^{1}$ \\ ${ }^{1}$ Institute für Festkörperforschung, Forschungszentrum Jülich, D-52425 Jülich, Germany \\ ${ }^{2}$ Institut für Theoretische Physik II, Heinrich-Heine-Universität Düsseldorf, D-40225 Düsseldorf, Germany
}

(Received 3 July 2003; published 15 December 2003)

\begin{abstract}
Adsorption of monovalent and multivalent cations and anions on a deoxyribose nucleic acid (DNA) molecule from a salt solution is investigated by computer simulation. The ions are modeled as charged hard spheres, the DNA molecule as a point charge pattern following the double-helical phosphate strands. The geometrical shape of the DNA molecules is modeled on different levels ranging from a simple cylindrical shape to structured models which include the major and minor grooves between the phosphate strands. The densities of the ions adsorbed on the phosphate strands in the major and in the minor grooves are calculated. First, we find that the adsorption pattern on the DNA surface depends strongly on its geometrical shape: counterions adsorb preferentially along the phosphate strands for a cylindrical model shape, but in the minor groove for a geometrically structured model. Second, we find that an addition of monovalent salt ions results in an increase of the charge density in the minor groove while the total charge density of ions adsorbed in the major groove stays unchanged. The adsorbed ion densities are highly structured along the minor groove while they are almost smeared along the major groove. Furthermore, for a fixed amount of added salt, the majorgroove cationic charge is independent of the counterion valency. For increasing salt concentration the major groove is neutralized while the total charge adsorbed in the minor groove is constant. DNA overcharging is detected for multivalent salts. Simulations for larger ion radii, which mimic the effect of ion hydration, indicate an increased adsorbtion of cations in the major groove.
\end{abstract}

DOI: 10.1103/PhysRevE.68.061903 PACS number(s): 87.15.Aa, 87.10.+e, 82.70.Dd, 61.20.Ja

\section{INTRODUCTION}

In addition to its biological role as the carrier of genetic information [1], deoxyribose nucleic acid (DNA) in solution exhibits typical polyelectrolyte behavior. Its physicochemical properties, such as melting temperature, transition between different DNA forms, and binding interaction of proteins and other ligands, strongly depend on the added salt concentration in aqueous solution. Some physical properties of DNA, such as its osmotic pressure and activity coefficients [2], can be explained within a simple polyelectrolyte picture of DNA as obtained via the counterion condensation theory of Manning [3], Poisson-Boltzmann (PB) theory [4-7], and Monte Carlo simulations $[4,8-12]$ of a homogeneously charged cylinder. Although a cylinder with an effective homogeneous line charge density might be an appropriate model for studying properties far away from the DNA surface, more details become relevant as one approaches the DNA surface. These details can be classified in the following way. First the actual charge distribution along the DNA molecule is not a homogeneous line charge but a discrete set of phosphate charge groups along a double helix. Next, the geometric shape of the DNA including the major and minor grooves between the two phosphate strands becomes relevant. Finally, molecular details of the DNA surface should be considered, including specific interactions and explicit solvent molecules.

The PB calculations have been successfully applied to investigate DNA electrostatics, such as the electrostatic field of a double-helix charge distribution [13] and of all-atom DNA models $[14,15]$. A comparison between the simulation and $\mathrm{PB}$ results for the multivalent counterion distribution around DNA, addressed in Refs. [4,10,15-17], reveals great differences between them. The reason for such differences is the lack of the hard core and Coulomb correlations in meanfield-based PB theory. On the other hand, an all-atom DNA simulation in solution is computationally very costly and can be performed only with small system sizes and low salt concentrations [18-28]. Therefore we focus in this paper on a "primitive approach" [29] which goes beyond the simplistic model of a line charged cylinder but still does not include full atomic details. The physical reason to do so is that most of the general properties of DNA are expected to result from a combination of Coulomb and excluded volume interactions which are the dominant parts of the total interactions for long and short ranges [30]. Following this strategy, we disregard the discrete structure of water, replacing it by a dielectric background, but treat the salt ions, the double-helical charge pattern on the DNA molecule, and the geometrical grooved shape of the DNA molecule explicitly.

This paper focuses on the cation and anion adsorption pattern on the DNA surface. In particular, in contrast to earlier simulations which present only rotationally averaged data for adsorbed ions [19,31-36], we resolve the adsorption in the major and minor grooves and on the phosphate strands. Both a qualitative and a quantitative knowledge of the adsorption pattern is desirable, since it is needed as a crucial input in other more coarse-grained approaches like the Kornyshev-Leikin theory of DNA-DNA interaction [37]. It is known that the details of the adsorption pattern strongly influence the effective interaction forces and even govern the sign of the interaction. Hence the adsorption pattern will have direct consequences for the aggregation and bundling of DNA molecules caused by an effective mutual attraction [38-40]. It is also known that adding multivalent ions to the solution causes drastic changes in the ion adsorption and in the DNA aggregation and bundling [41-43]. Many experi- 
mental facts have been collected regarding the adsorption of multivalent ions on the DNA. To name just a few, there is experimental evidence indicating that $\mathrm{Mn}^{2+}$ and $\mathrm{Cd}^{2+}$ condense on DNA $[44,45]$ but $\mathrm{Ca}^{2+}$ and $\mathrm{Mg}^{2+}$ do not $[45,46]$. This points to an important specificity [47-51] and the question arising is whether this can be understood in simple terms of effective ion radii. Multivalent counterions of valency larger than 2, on the other hand, such as trivalent spermidine and tetravalent spermine are believed to play a key role in maintaining cellular DNA in a compact state [52-55]. The compactification of DNA $[39,40]$ seems to be mediated by their adsorption on the DNA surface [39,56,57]. Therefore there is a need to study the role of high-valency counterions in the DNA adsorption pattern in a systematic way.

While the Manning condensation theory on a homogeneously charged cylinder is well studied by now [58], it is $a$ priori unclear how the adsorbed counterions will partition themselves in the two grooves and on the phosphate strands. Moreover, the adsorption of anions that carry the same charge as the phosphates in the major grooves is an interesting issue, in particular for higher counterion valencies.

The goal of this paper is twofold. First, we aim to predict both qualitatively and quantitatively the nature of the adsorption pattern on a single DNA molecule for a given added salt concentration and given microion valencies and microion radii. Ion-specific effects, however, enter only via the ion sphere radius and its charge. Although the actual numbers may be influenced by further details such as the dielectric properties of water at close ion distances, we think that the trends of our findings about a change in ion valency and salt concentration will be robust. In particular, for high-valency ions, the interactions should be dominated by the Coulombic part such that our "primitive" model should be more appropriate. Second, on a more technical level, we would like to investigate the influence of the geometrical structure of the DNA shape used in the theoretical model of the adsorption pattern. It is expected that a grooved shape will attract more counterions into the grooves electrostatically, such that the adsorption pattern will depend on the geometrical shape used in the model.

In our computer simulations we find that the adsorption pattern on the DNA surface depends strongly on the geometrical model shape of the DNA surface. In detail, three different shapes, modeling the geometrical structure of the grooves on different levels, are considered. It is found that counterions adsorb preferentially along the phosphate strands for a cylindrical model shape but in the minor groove for a geometrically structured model.

Furthermore, we find that addition of more monovalent salt ions, provided the counterions are also monovalent, results in an increase of the charge density in the minor groove, while the total charge density of ions adsorbed in the major groove stays unchanged. The adsorbed ion densities are highly structured along the minor groove while they are almost smeared along the major groove.

We also analyze the influence of the ion valency on the ion adsorption pattern on the DNA surface. We show that for any fixed amount of salt the major-groove cationic charge is constant for any counterion valency. For added multivalent salt, we show the existence of a major-groove neutralization phenomenon while the minor-groove total charge remains constant.

We also address the DNA overcharging phenomenon, which is of special interest in biology, for example, for the delivery of genes to the living cell for the purpose of gene therapy. Since both the bare DNA and cell surface are negatively charged, normally DNA does not approach the cell surface. An overscreened DNA molecule, however, is effectively positively charged such that it could pass through the negative cell membrane. Our simulations of show that the overcharging of DNA appears generally in multivalent salt solution regardless of the counterion valency. Finally, we performed a few simulations with larger ion radii in order to mimic a larger ion hydration shell in the solvent. Our findings show that for an increasing ion radius more cations go to the major groove, whereas the minor-groove and strand ionic occupations shrink.

The paper is organized as follows. In Sec. II we discuss different models for the DNA shape. Our simulation technique and model parameters are presented in Sec. III. Results are given in Sec. IV. Finally, we conclude in Sec. V.

\section{DIFFERENT MODELS FOR THE DNA SHAPE}

We consider the $B$ form of DNA, which is the most common state of DNA in aqueous solutions. It has an inner core formed by nucleotide pairs, and two sugar-phosphate strands spiraling around the core. The latter forms a well-known double helix with a pitch length of about $34 \AA$ and a core radius of about $9 \AA$. There are two phosphate groups per base pair and ten base pairs per pitch (or helical turn). The axial rise per base pair along the DNA long axis is $3.4 \AA$; hence there is one elementary charge per each $1.7 \AA$. The average value of the angle between the adjacent base pairs is $36^{\circ}$ which makes the average distance between neighboring charges on the DNA surface $7 \AA$. This distance, which is much smaller than the helical pitch, is of the order of the Debye screening length under physical conditions. Finally, the helix persistence length is about $500 \AA$.

Three different models for the DNA shape will be studied here: (i) a simple cylinder model (CM), (ii) an extended cylinder model (ECM) with a grooved structure, and (iii) the Montoro-Abascal model (MAM) [33]. The cross sections of these DNA models in the $x y$ plane that is perpendicular to the DNA long axis ( $z$ axis) and hits two phosphates on different strands are sketched in Fig. 1 and will be discussed subsequently; in all three models the phosphate charges are discretely placed at certain positions coincident with those of the phosphorus atoms in crystalline DNA.

(i) Cylindrical model; see Fig. 1(a). This model was used by Kornyshev and Leikin [37] and by two of us in another study [59]. In the CM, the cylindrical DNA core possesses a diameter of $D=20 \AA$. Two strings of pointlike and monovalent phosphate charges of size $d_{p}=0.4 \AA$ have cylindrical coordinates $\left(\rho_{i}^{s}, \phi_{i}^{s}, z_{i}^{s}\right)$ relevant to the phosphate sites of the $B$ form of DNA:

$$
\rho_{i}^{s}=D / 2=10 \AA, \quad \phi_{i}^{s}=\phi_{0}^{s}+i \times 36^{\circ}, \quad z_{i}^{s}=z_{0}^{s}+i \times 3.4 \AA .
$$

Here $s=1,2$ specifies the nucleic acid strand, $i=0, \ldots, 9$ 

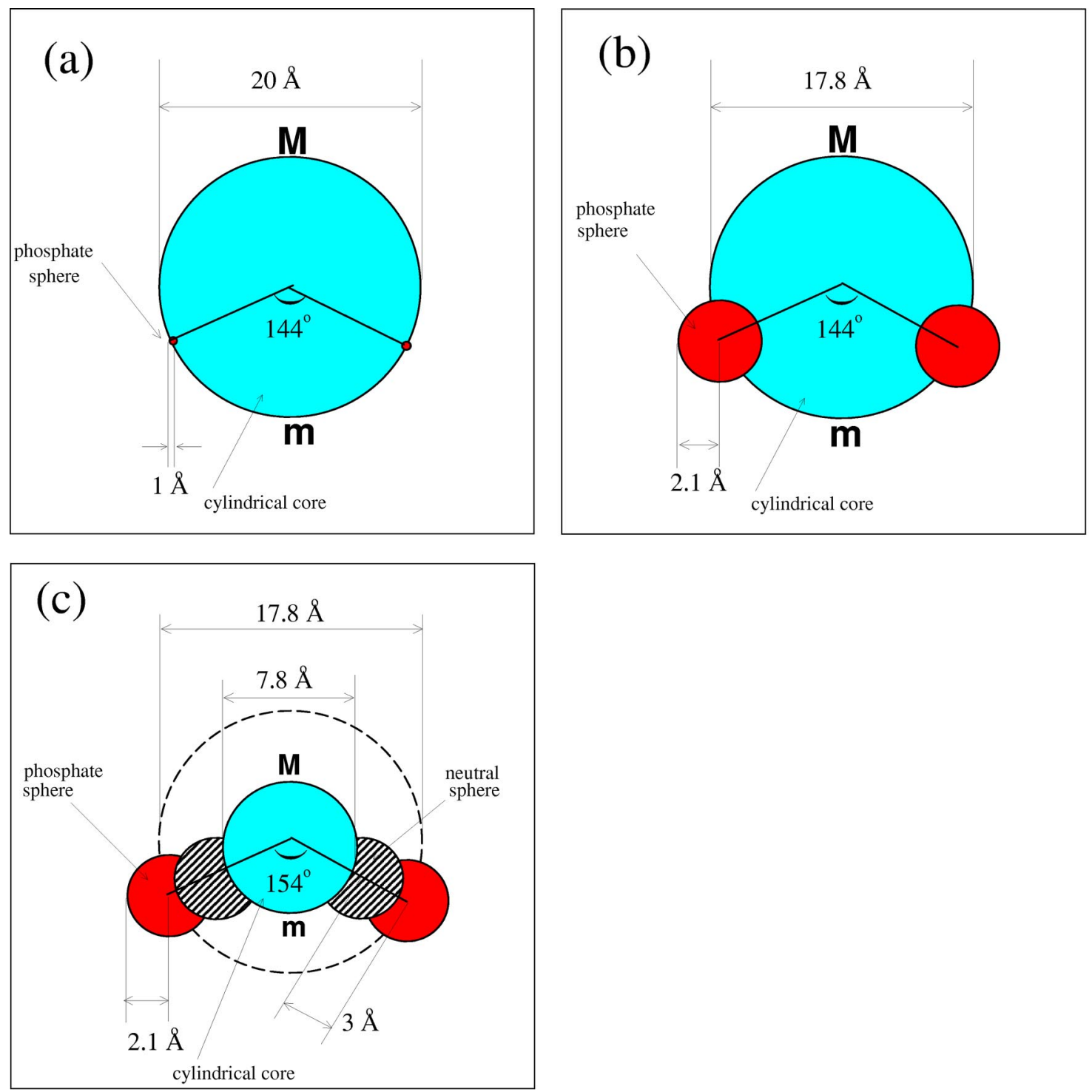

FIG. 1. Cross sections of different DNA models in the $x y$ plane. (a) Cylinder model, (b) extended cylinder model, (c) grooved or Montoro-Abascal-like model. Phosphate charges are shown as dark spheres. The DNA cylindrical core is colored in gray; the hatched areas correspond to neutral hard spheres. The inscribed letters " $\mathrm{M}$ " and " $\mathrm{m}$ " denote the major and minor grooves, respectively.

describes a full DNA turn, and the $z$ axis is the long DNA axis. Furthermore, $\phi_{0}^{1}=0^{\circ}, z_{0}^{1}=0$ for the first strand ( $s$ $=1)$ and $\phi_{0}^{2}=144^{\circ}, z_{0}^{2}=0$ for the second strand $(s=2)$.

(ii) Extended cylinder model; see Fig. 1(b). As designed by Lyubartsev and Nordenskiöld [31], both the helical DNA grooves and the discrete charge localization on the DNA surface are incorporated. In the ECM, the DNA molecule has a hard cylindrical core of diameter $D=17.8 \AA$, which is slightly smaller than in the $\mathrm{CM}$. The phosphate charges are swollen to hard spheres of diameter $d_{p}=4.2 \AA$ explicitly forming grooves. Other DNA parameters are similar to those of the CM.

(iii) Montoro-Abascal model; see Fig. 1(c). This more elaborate model was first introduced in Ref. [33]; the grooved structure of DNA is increased by adding another neutral sphere between the cylindrical core and the charged phosphate sphere. In detail, the inner DNA cylindrical core of $D=7.8 \AA$ is overwound by two strings of overlapping spheres. The outer string of monovalently charged phosphate spheres is centered at a radial coordinate of $8.9 \AA$. The radial position of the inner string of neutral spheres is $5.9 \AA$. Both spheres have the same $\phi$ and $z$ coordinates and diameter $d_{p}=4.2 \AA$ to incorporate a grooved geometry for the DNA molecule. Clearly, such a design of an overlapped cylinder and two spheres creates a more grooved DNA profile with a deeper cavity in the center of the minor groove. For other details of the MAM and its reliability, we refer the reader to the original papers [33-35].

\section{SIMULATION TECHNIQUE AND SYSTEM PARAMETERS}

In our simulation setup, the $B$-DNA molecule is located in the center of a cubic simulation box. The cylinder axis is 
parallel to the $z$ axis and crosses the $x y$ plane at position $\vec{R}(L / 2, L / 2,0)$. The size $L$ of the simulation box was chosen to be $L=102 \AA$, corresponding to three full turns of $B$-DNA with a pitch length $P=34 \AA$ and with $N_{p}=3 \times 20=60$ phosphate groups along the DNA [34]. There is a small shift in the $z$ coordinate of two discrete phosphate charges belonging to two different helices of $\Delta z=0.78 \AA$.

Periodic boundary conditions are applied in all three directions; hence the DNA replicas in the $z$ direction produce an infinitely long DNA molecule, and an infinite array of DNA replicas in neighboring cells is simulated. The phosphate spheres are monovalent, i.e., their charge $q_{p}<0$ corresponds to one elementary charge $|e|, q_{p}=-|e|$, and they have an effective diameter $d_{p}$ which is a variable parameter in our different shape models. In addition to the DNA phosphates, the system contains $N_{c}$ counterions of concentration $C_{c}=N_{c} / V^{\prime}$ with charge $q_{c}$, and $N_{s} \equiv N_{+} \equiv N_{-}$pairs of salt ions of concentration $C_{s}=N_{s} / V^{\prime}$ with charges $q_{+}$and $q_{-}$. Here $V^{\prime}$ is the free volume in the simulation box accessible for these small ions, where the excluded volume of the DNA molecule has been subtracted. The counterion number $N_{c}$ in the simulation box is fixed by the charge of the DNA molecule due to the constraint of global charge neutrality, $N_{c} q_{c}$ $=60\left|q_{p}\right|$. For simplicity, we shall always deal with a symmetric salt case, $\left|q_{+}\right|=\left|q_{-}\right|$. All small ions are modeled as hard spheres of (hydrated ion) diameter $d_{c}$. For most of our simulations, $d_{c}=3 \AA$, but we have obtained data for larger ion sizes $d_{c}=6 \AA$ and $d_{c}=8 \AA$ as well. The whole system is held at room temperature $T=298 \mathrm{~K}$. The dielectric constant $\epsilon=78$ of the solvent is assumed to be uniform throughout the suspension (the same value inside the DNA molecule and in the suspending medium), which avoids electrostatic images.

The interactions between the mobile ions and the phosphate charges are described within the framework of the primitive model as a combination of excluded volume and Coulomb interactions reduced by the dielectric constant $\epsilon$ of the solvent. The corresponding pair interaction potential between the different charged hard spheres is

$$
V_{i j}(r)= \begin{cases}\infty & \text { for } r \leqslant\left(d_{i}+d_{j}\right) / 2, \\ \frac{q_{i} q_{j} e^{2}}{\epsilon r} & \text { for } r>\left(d_{i}+d_{j}\right) / 2,\end{cases}
$$

where $r$ is the interparticle separation and $i, j$ are indices denoting the different particle species. Possible values for $i$ and $j$ are $c$ (for counterions),,+- (for positively and negatively charged salt ions), and $p$ (for phosphate groups). In addition, there is an interaction potential $V_{i}^{0}$ between the DNA hard cylinder and the free ions $i=c,+,-$ which is of simple excluded volume form such that these ions cannot penetrate into the cylinder. A similar excluded volume potential exist for the inner neutral DNA spheres in the elaborated MAM. Finally, the ionic strength $I$ and the Debye screening length $\lambda_{D}$ of the solution are defined as $I=\frac{1}{2}\left(q_{c}^{2} C_{c}\right.$ $\left.+\sum_{j=+,-} q_{j}^{2} C_{s}\right)$ and $\lambda_{D}=\sqrt{\epsilon k_{B} T / 4 \pi I}$. In order to compute the statistical averages over the mobile microions, we have performed conventional $N V T$ molecular dynamics simulations, where the long-range electrostatic forces were treated

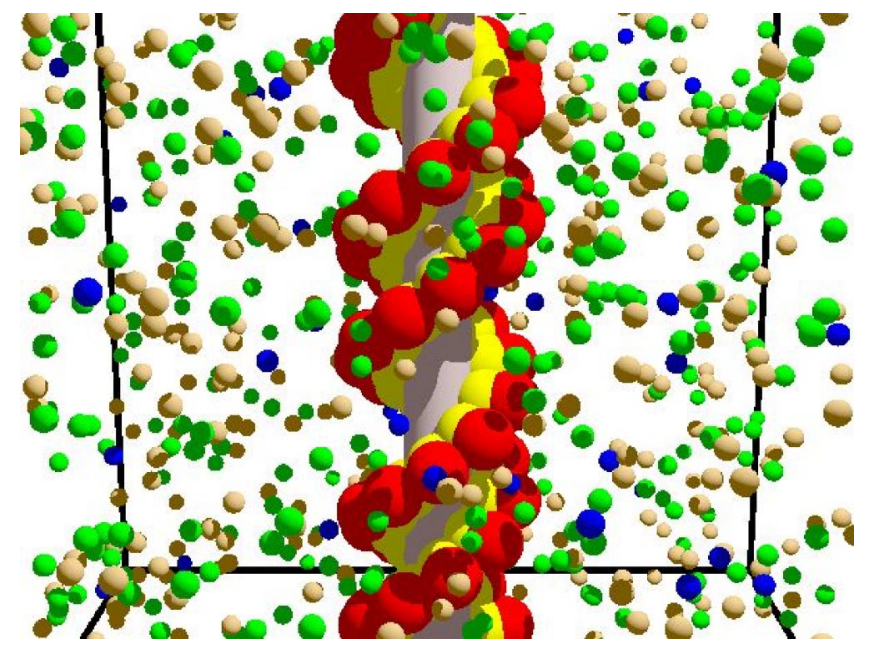

FIG. 2. Snapshot of the simulation box. The DNA molecule is drawn according to the MAM. Black spheres on the DNA strands represent the phosphate charges. Internal gray spheres between the phosphates and the DNA cylindrical core are neutral. Positive (negative) salt ions spread across the simulation volume are shown as open (hatched) spheres.

according to the Lekner procedure $[59,60]$. A typical simulation snapshot of the system is given in Fig. 2.

Our major goal is to calculate the mobile ion number densities $\rho_{j}(\vec{r})(j=c,+,-)$ around the DNA molecule. They are defined as a statistical average,

$$
\rho_{j}(\vec{r})=\left\langle\sum_{i=1}^{N_{j}} \delta\left(\vec{r}-\vec{r}_{i}^{j}\right)\right\rangle .
$$

Here $\left\{\vec{r}_{i}^{j}\right\}$ denote the positions of the $i$ th particle of species $j$. The canonical average $\langle\cdots\rangle$ over an $\left\{\vec{r}_{i}^{j}\right\}$-dependent quantity $\mathcal{A}$ is defined via the classical trace

$$
\begin{aligned}
\langle\mathcal{A}\rangle= & \frac{1}{\mathcal{Z}}\left\{\prod_{k=1}^{N_{c}} \int d^{3} r_{k}^{c}\right\}\left\{\prod_{m=1}^{N_{+}} \int d^{3} r_{m}^{+}\right\}\left\{\prod_{n=1}^{N_{-}} \int d^{3} r_{n}^{-}\right\} \\
& \times \mathcal{A} \exp \left(-\beta \sum_{i=c,+,-}\left[V_{i}^{0}+\sum_{j=c, p,+,-} U_{i j}\right]\right) .
\end{aligned}
$$

Here $\beta=1 / k_{B} T$ is the inverse thermal energy ( $k_{B}$ denoting Boltzmann's constant) and

$$
U_{i j}=\left(1-\frac{1}{2} \delta_{i j}\right) \sum_{l=1}^{N_{i}} \sum_{k=1}^{N_{j}} V_{i j}\left(\left|\vec{r}_{l}^{i}-\vec{r}_{k}^{j}\right|\right)
$$

is the total potential energy of the counter- and salt ions provided the phosphate groups are at positions $\left\{\vec{r}_{n}^{p}\right\}(n$ $\left.=1, \ldots, N_{p}\right)$. Note that the periodically repeated particles are incorporated implicitly in the interaction energy. Finally, the prefactor $1 / \mathcal{Z}$ in Eq. (3) ensures a correct normalization, $\langle 1\rangle=1$.

In computer simulations and different theoretical approaches the distance below which the ions are considered to be condensed is usually assumed to be in the range of one or 


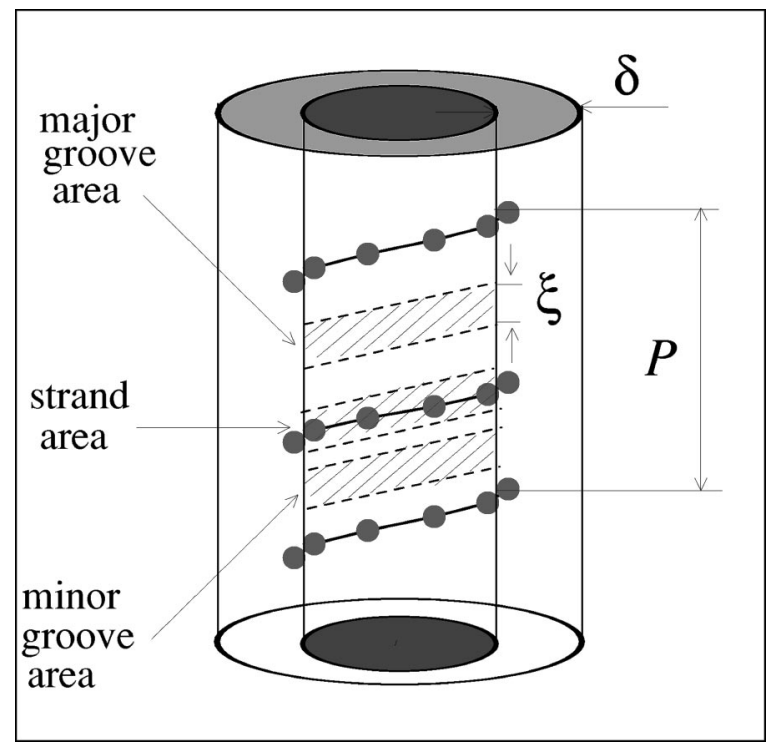

FIG. 3. A schematic picture to explain the procedure of ion density calculations along one pitch length $(P)$ of a DNA molecule. The filled circles connected with full lines are phosphate groups. The shaded areas correspond to a path along the major groove, minor groove, and one of the phosphate strands. The path height is $\xi=3.4 \AA$ and width is $\delta=5 \AA$.

two water molecule diameters. Thus the width of the condensation shell near the DNA surface is around $7 \AA$ in Manning theory [3], whereas a value of $5 \AA$ was invoked in other papers $[19,31,61,62]$. These values are larger than the thickness of the Stern layer $d_{l}=A / 4 \pi \lambda_{B}=2 \AA$ including ions bound to the molecular surface. Here $\lambda_{B}=e^{2} / \epsilon k_{B} T$ is the Bjerrum length and $A$ is an average area per elementary charge on the molecular surface. In our study we follow the latter criterion and treat the ions as condensed if the surfaceto-surface distance between the ion sphere and the DNA hard surface is not larger than $2 \AA$. In other words, we are interested in the population in special areas of the DNA surface of small ions rather than the actual ion condensation on the DNA surface [63]. In order to resolve the adsorbed ions along the strands and in the major and minor grooves, we integrate the ion density fields as given by Eq. (2) over a small volume close to the DNA surface. This volume is bounded by the parallel surface to the DNA surface at distance $\delta=2 \AA+d_{c} / 2$ and has a height $\xi$ in the $z$ direction. The volume follows the helical symmetry of the DNA molecule. A schematic view of this condensation shell and the definition of the groove and strand adsorption paths around the DNA are given in Fig. 3. We have separately counted cations, which are comprised of counterions and positively charged salt ions, and anions (coions). The angular-resolved cation and anion density profiles along the phosphate strands and the minor and major grooves represent the main results of this paper. We call a plot of the densities versus polar angle a "panoramic" view of the density profiles and shall present exhaustive data for these quantities for different parameters in the next section. Additionally, we define the charge densities of adsorbed ions by $\rho^{(+)}=\Sigma_{j=c,+} q_{j} \rho_{c}^{(j)}$ and $\rho^{(-)}=q_{-} \rho_{c}^{(-)}$, where $\rho_{c}^{(j)}$ are the number densities of the
TABLE I. Parameters used for the different simulation sets.

\begin{tabular}{lcc}
\hline \hline Set & $q_{c}$ & $q_{s}$ \\
\hline Set 1 & 1 & 1 \\
Set 2 & 1 & 2 \\
Set 3 & 3 & 2 \\
Set 4 & 2 & 1 \\
Set 5 & 3 & 1 \\
Set 6 & 2 & 2 \\
\hline \hline
\end{tabular}

adsorbed ions of species $j \in\{c,+,-\}$.

Our molecular dynamics (MD) simulations cover a broad range of salt concentrations from 0.1 to $1.61 \mathrm{~mol} / \mathrm{l}$, where the latter corresponds to 2000 salt ions of both charges in the simulation box. The counterion concentration is $C_{c}$ $=0.1 / q_{c} \mathrm{~mol} / \mathrm{l}$. The time step $\Delta t$ of the simulation was typically chosen to be $5 \times 10^{-3} \sqrt{m D^{3} / e^{2}}$, with $m$ denoting the (fictitious) mass of the mobile ions. This corresponds to an average ion displacement of $0.03 \AA$ per time step such that the reflection of counterions following the collision with the combined surface of DNA is calculated with high precision. For every run the state of the system was checked during the simulation time. This was done by monitoring the temperature, average velocity, distribution function of velocities, and total potential energy of the system. On average it took about $5 \times 10^{4}$ MD steps to get to equilibrium. Then during $5 \times 10^{4}-5 \times 10^{6}$ time steps we gathered statistics to perform the canonical averages for the calculated quantities. Details of the simulated states are summarized in Table I. During the simulation we checked that there was a continuous exchange of the adsorbed and "free" ions, which demonstrates that our systems are in equilibrium.

\section{RESULTS}

\section{A. Monovalent ions}

Let us first discuss monovalent ions. For the extended cylindrical model, a panoramic angular cation and anion distribution over $0<\phi<2 \pi$ is plotted in Fig. 4 for the system parameters $q_{c}=1, q_{s}=1$ (set 1 of Table I) and $C_{s}$ $=0.1 \mathrm{~mol} / \mathrm{l}$. The cations cluster in front of the charged phosphates, almost in a site-binding-like manner [32-35], showing a strong structuring, while the minor-groove cation density is less structured. The smallest cation density is in the major groove. The anion densities in the DNA grooves and on the phosphate strands are considerably smaller and not structured at all. The major-groove population of anions is higher than that in the minor groove and on the strands. The same quantities are shown for the cylinder model and the Montoro-Abascal model in Figs. 5 and 6 respectively. As compared to the ECM, an increase of condensed cations along the phosphate strands at the expense of their accumulation in the minor and major grooves is clearly visible for the CM. In the most realistic MAM, however, more cations bind and locate in the DNA grooves $[64,65]$ at the expense of their accumulation on strands. Such an ion relocation from the strands into the grooves entails an entropy gain for salt 


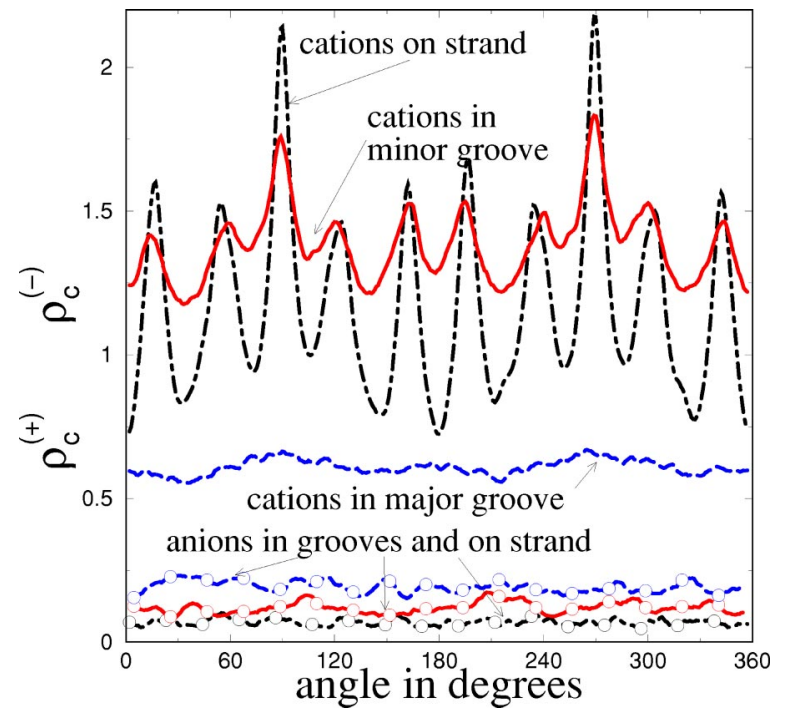

FIG. 4. Panoramic view of the condensed small ion densities near the DNA surface for $q_{c}=1, q_{s}=1$ (set 1), $C_{s}=0.1 \mathrm{~mol} / \mathrm{l}$, and the ECM. The density unit is $0.22 \mathrm{~mol} / \mathrm{l}$. Dot-dashed lines: distribution on the phosphate strands; full lines: distribution in the minor groove; dashed lines: distribution in the major groove. Lines without or with symbols correspond to cation $\rho_{c}^{(+)}$or anion $\rho_{c}^{(-)}$densities. The value of the cation distribution is much larger than the anion distribution (where the latter is enhanced by a factor of 10).

ions. In the MAM the population of condensed cations along the strands is less by a factor of 2 as compared to the structured minor-groove density. Our conclusion arising from Figs. 4, 5, and 6 is twofold. First, on a technical level, the inclusion of a grooved shape in the excluded volume of the DNA molecule is crucial for ion adsorption. It completely changes the charge and structure of the adsorption pattern. Second, taking the MAM as the most realistic description of the DNA shape, we can conclude that adsorbed cations ex-

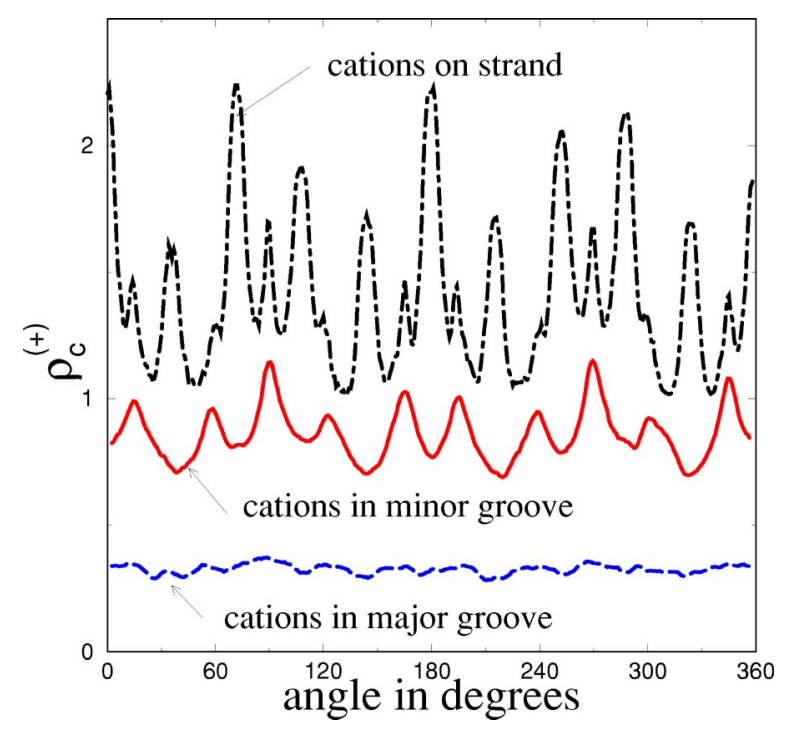

FIG. 5. Panoramic view of the condensed small ion densities near the DNA surface for the cylinder model. The parameters are the same as in Fig. 4.

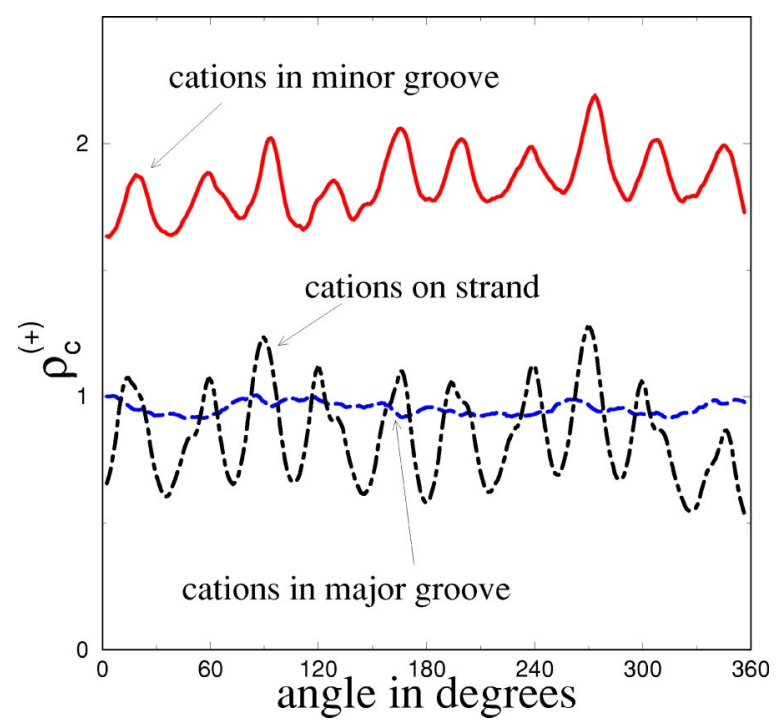

FIG. 6. Panoramic view of the condensed small ion densities near the DNA surface for the Montoro-Abascal model. The parameters are the same as in Fig. 4.

hibit a pronounced spatial structure along the minor groove. This strong structuring of cations in the minor groove will also induce a structuring of the water molecules in the minor groove and might therefore be related to the so-called "spine of hydration in the minor groove" which is attributed to a high water ordering there $[18,66-70]$. In general, the spine of hydration emerges due to the occasional intrusion of counterions in the particularly electronegative regions in the minor groove [71] and is addressed in Refs. $[26,34,48,49,69,72-74]$. We think that the experimentally measured hydration pattern can be a fingerprint of cation ordering in the minor groove $[68,75,76]$.

The dependence of the adsorption pattern on the salt concentration is shown in Fig. 7. The added monovalent salt concentration $C_{s}$ is increased from $0.2 \mathrm{~mol} / \mathrm{l}$ to $1.61 \mathrm{~mol} / \mathrm{l}$ for $q_{c}=1, q_{s}=1$ (set 1). The highest cationic occupation is again in the minor groove but more anions condense in the major groove as the salt density is increased [35]. The total major-groove charge, calculated as the difference between the cation (dashed line) and anion (dashed line with symbols) densities in the major groove, is almost independent of the amount of added salt (see the single arrows in Fig. 7). In other words, for any monovalent salt density the geometry of the major groove and the electrostatic field of the two adjacent phosphate strands regulate the cation and anion populations and keep the major-groove charge unchanged. On the other hand, the minor groove is positively charged as the bulk salt density increases (see the difference between the full line and the full line with symbols for different salt densities in Fig. 7). The visible cation structuring in the major groove with dense salt (see the right side of Fig. 7), is consistent with the experimental evidence for recurring hydration patterns in the major groove $[74,77,78]$. The other observation is the constancy of the gap between the minor- and major-groove cationic occupancies shown in Fig. 7 by the double arrows for different salt densities. Obviously, in solutions where the DNA phosphate charges are effectively 


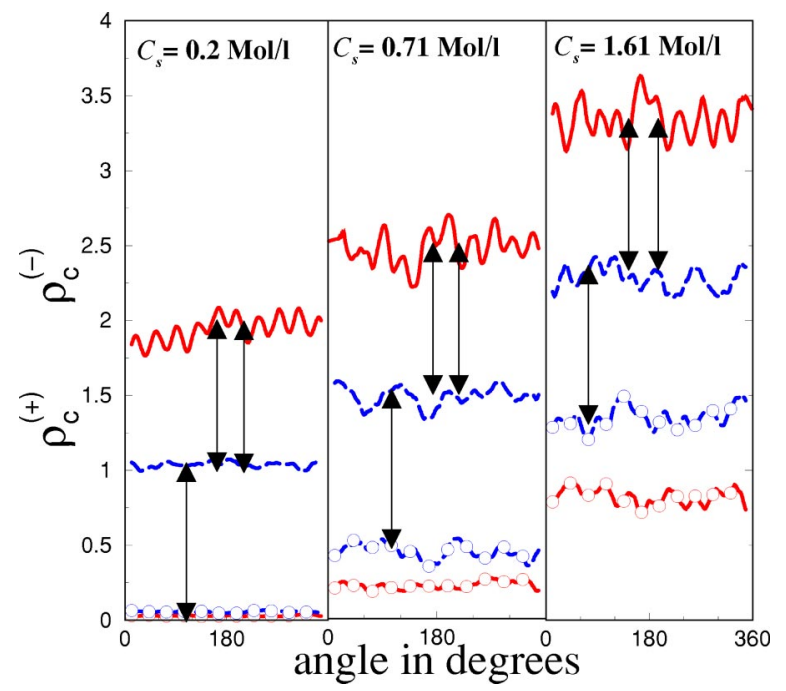

FIG. 7. Panoramic view of ion densities in DNA grooves for MAM and $q_{c}=1, q_{s}=1$ (set 1). Three full panoramic views along the $x$ axis correspond to three different salt densities; from left to right, $C_{s}=0.2 \mathrm{~mol} / 1,0.71 \mathrm{~mol} / 1,1.61 \mathrm{~mol} / \mathrm{l}$. Full line: charge distribution in the minor groove; dashed line: charge distribution in the major groove. Lines without or with symbols correspond to cation $\rho_{c}^{(+)}$or anion $\rho_{c}^{(-)}$densities. The single arrow indicates the constancy of the major-groove charge at different added salt densities. The constancy of the difference between the cationic charges of DNA grooves is shown as a double arrow.

screened out, it is the osmotic pressure of salt that pushes ions close to the DNA surface. We note that the constancy of the accumulated charge in the major groove and the constancy of the difference between cationic populations of major and minor grooves do not appear in the ECM and CM. This result implies the crucial role of the DNA shape in the ion distribution around the DNA molecule. Thus, the MAM, which can be viewed as a "soft" cylinder compared to the ECM and CM, provides more "grooved" space for the small ions. Hence the ions "captured in these grooves," together with the phosphates, actively regulate the groove occupancies as more salt is added.

We finally remark that throughout all runs it was revealed that at a distance 5-7 $\AA$ away from the DNA surface a cylindrical symmetry of radial ion distribution is completely restored in accordance with the observations of Refs. $[31,34,36,79]$. Thus the effect of the discreteness of DNA charges on counterion concentration profiles is generally small and disappears a few angstroms away from the DNA surface $[80,81]$.

\section{B. Multivalent counterions and monovalent salt}

We now consider the case of multivalent counterions and a monovalent salt, for which the Coulomb correlations between the counterions and the DNA phosphates are strong. We keep the salt concentration fixed and increase the counterion valency. This leads to a higher on-strand adsorption of counterions, which implies less condensation in the minor and major groove [see Fig. 8(a)]. Also for high counterion
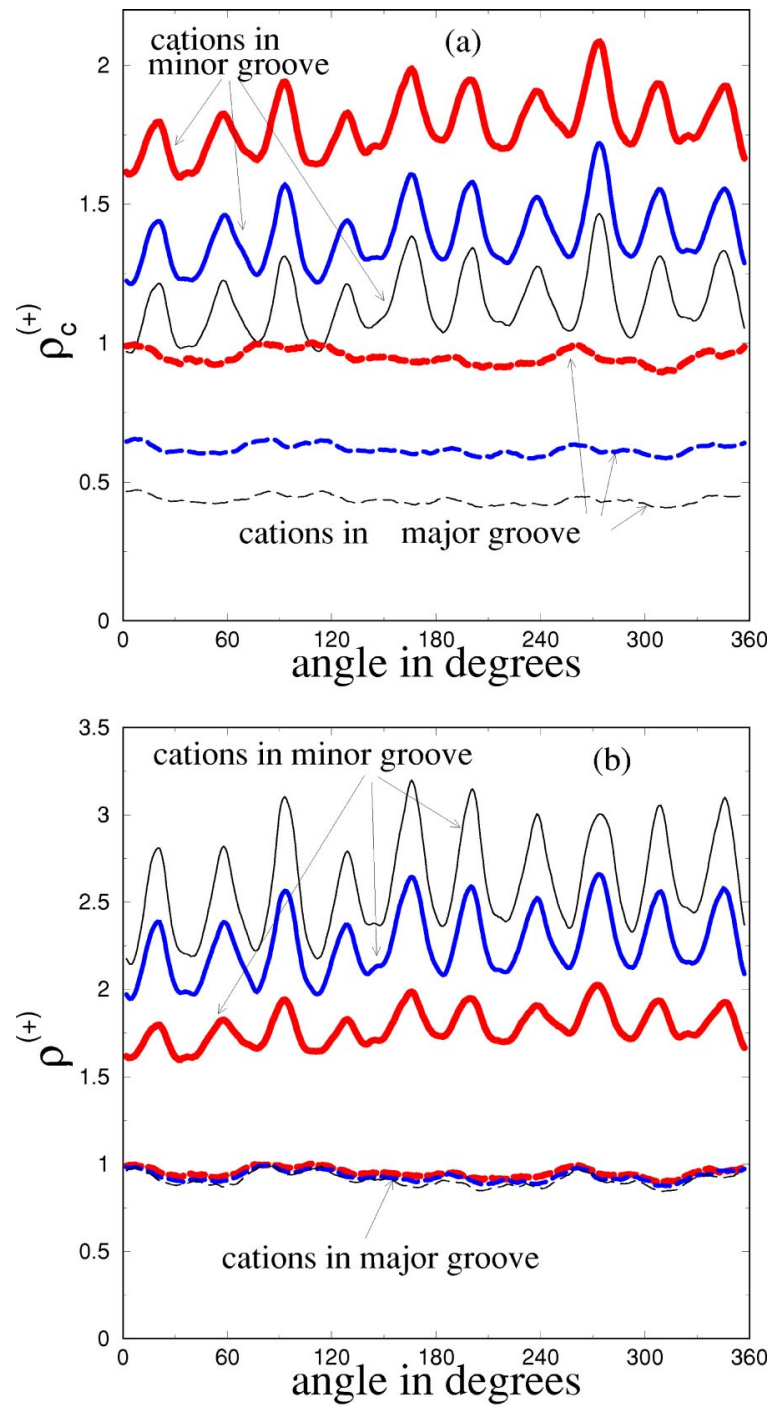

FIG. 8. Panoramic view of the cation number density $\rho_{c}^{(+)}$(a) and charge density $\rho^{(+)}$(b) for the MAM. Monovalent salt $C_{s}$ $=0.1 \mathrm{~mol} / 1$ and different counterion valencies. Full line: cations in the minor groove; dashed line: cations in the major groove. Thick lines: monovalent counterions (set 1); medium sized line: divalent counterions (set 4); thin line: trivalent counterions (set 5).

valencies, the cation adsorption on the minor groove is higher than that on the major groove in accordance with Ref. [82]. The same trends appear also in the ECM and in the CM.

Furthermore, for increasing counterion valency, Fig. 8(b) reveals that the total adsorbed charge in the major groove is almost constant while it is getting more positive in the minor groove. The increase of adsorbed cations in the minor groove causes a visible spatial structuring along the minor groove [see the surging oscillations in Figs. 8(a) and 8(b)]. Again, such an ion structuring is perhaps connected to the experimentally observed spine of hydration. Note that the number of adsorbed ions in the major groove drastically decreases in the CM and ECM for higher counterion valencies, which reaffirms the crucial role of the modelling of the DNA shape. 

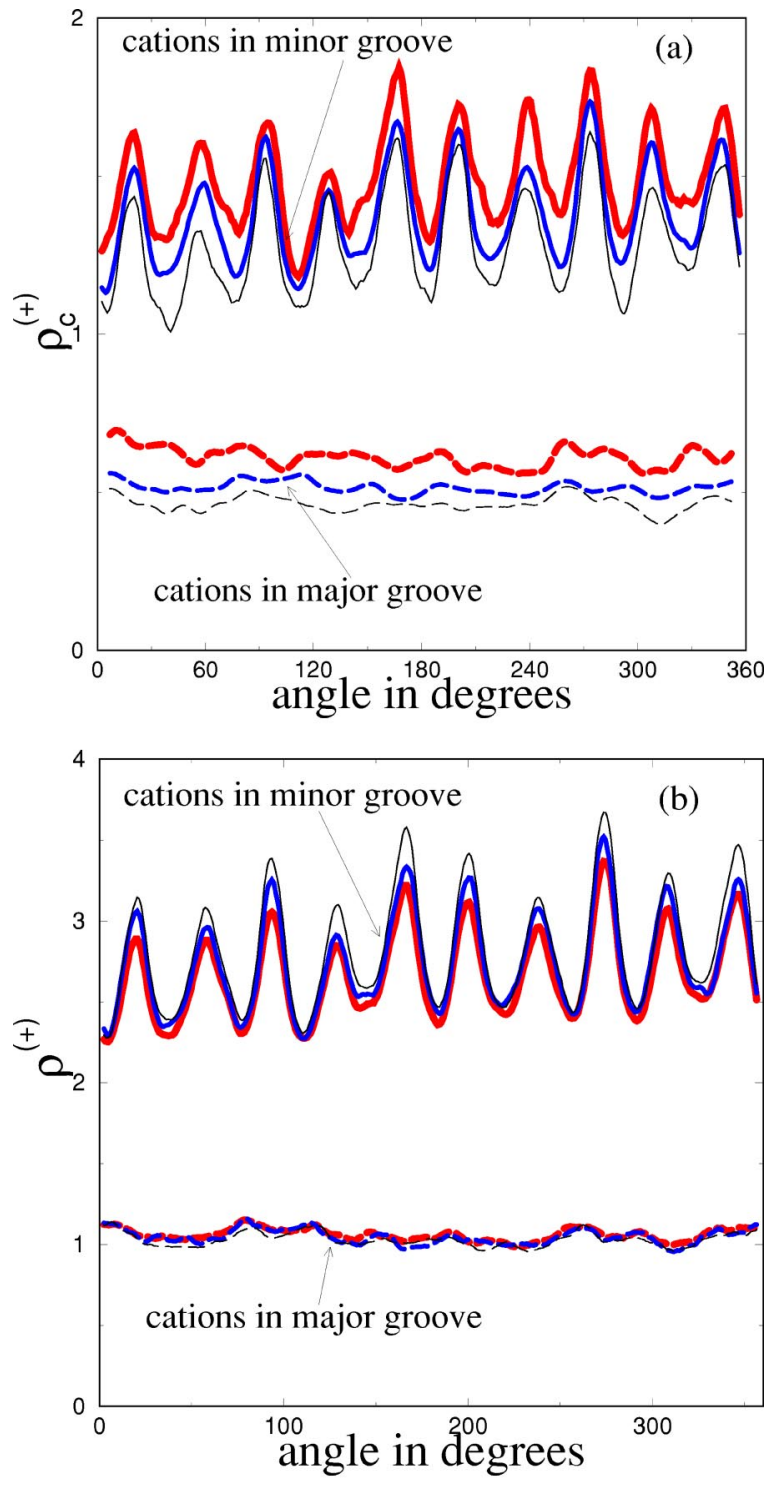

FIG. 9. Panoramic view of the cation number density $\rho_{c}^{(+)}$(a) and charge density $\rho^{(+)}$(b) for the MAM. Divalent salt $C_{s}$ $=0.2 \mathrm{~mol} / \mathrm{l}$ and different counterion valencies. Full lines: cations in the minor groove; dashed lines: cations in the major groove. Thick lines: monovalent counterions (set 2); medium sized line: divalent counterions (set 6); thin line: trivalent counterions (set 3).

\section{Multivalent counterions and multivalent salt}

For fixed divalent salt concentration and increasing counterion valency, similar results are obtained, as shown in Fig. 9. The number of adsorbed cations decreases in the grooves [see Fig. 9(a)] and the total charge in the major groove stays constant [very close to its value in Fig. 8(b)] while it is rising slightly in the minor groove [see Fig. 9(b)]. Hence we conclude that the total major groove charge is independent of the valencies of both cations and anions.

We now gradually increase the amount of added divalent salt. The in-groove ion distributions are shown in Fig. 10 for three different salt concentrations. There are two remarkable effects of increasing salt concentration. First the total charge of ions adsorbed in the major groove is approaching zero,

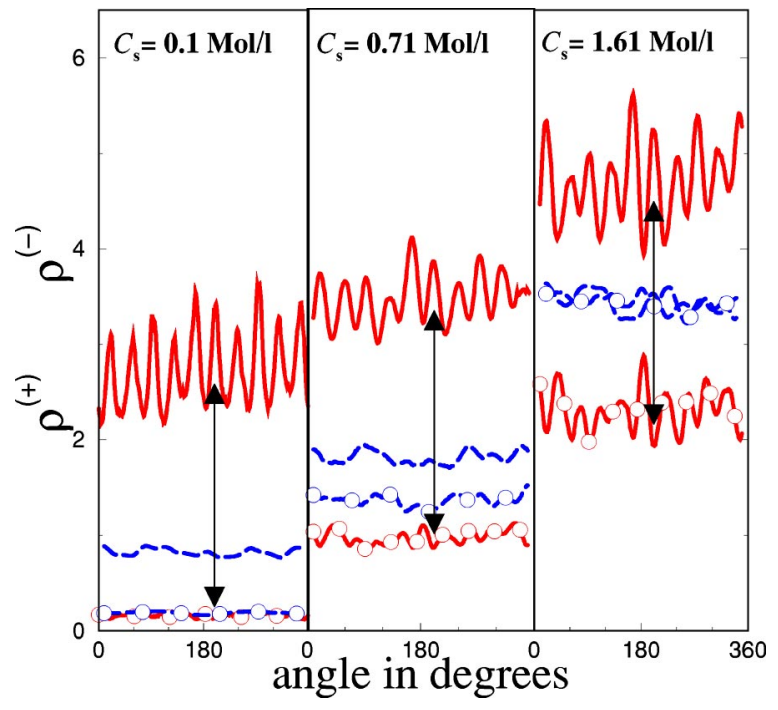

FIG. 10. Panoramic view of ion charge densities in the DNA grooves for the MAM, trivalent counterions, and divalent salt (set 3 ) and three different salt concentrations: from left to right, $C_{s}$ $=0.1 \mathrm{~mol} / 1,0.71 \mathrm{~mol} / 1,1.61 \mathrm{~mol} / 1$. Full line: charge distribution in the minor groove; dashed line: charge distribution in the major groove. Lines without or with symbols correspond to cation $\rho^{(+)}$or anion $\rho^{(-)}$charge densities. The shrinking of the gap between the major-groove cationic (dashed line) and anionic (dashed line with symbols) charges, as more salt is added, is the onset of the majorgroove neutralization. The minor-groove charge does not depend on the salt concentration; see arrows which indicate the total charge density in minor groove [the gap between the minor-groove cation (full line) and anion (full lines with symbols) charges].

i.e., the major groove is neutralized for high salt concentration. Second, the total charge in the minor groove is pretty robust against an increase in salt concentration (see the length of the arrows in Fig. 10). The effects of major groove neutralization are lost if a less realistic DNA shape is used as shown in Fig. 11 for the $\mathrm{CM}$ and the ECM.

In Fig. 12, the ion densities adsorbed on the strands are shown for trivalent counterions as a function of divalent salt concentration. The total charge adsorbed along the strands increases with added salt concentration. Together with the constancy of the minor groove and the neutralization of the major groove, this produces an overcharging effect of the DNA which is discussed in the next subsection.

\section{Overcharging effect}

Charge inversion (also known as an overcharging, overneutralization, or charge reversal) is possible for a variety of macroions, ranging from the charged surface, charged lipid membranes to colloids, DNA, and actin. It is believed that for this effect to occur the cations have to be multivalent to enhance nonlinear effects, such as Coulomb correlations. Thus, in the presence of multivalent ions, the ionic cloud may not only compensate the polyion charge, but even exceed it, resulting in opposite values of the electrostatic potential at some distances. Overcharging has been observed in Monte Carlo simulation [10,83-85], hypernetted chain calculations [86-88], and modified, PB theories with nonlinear 


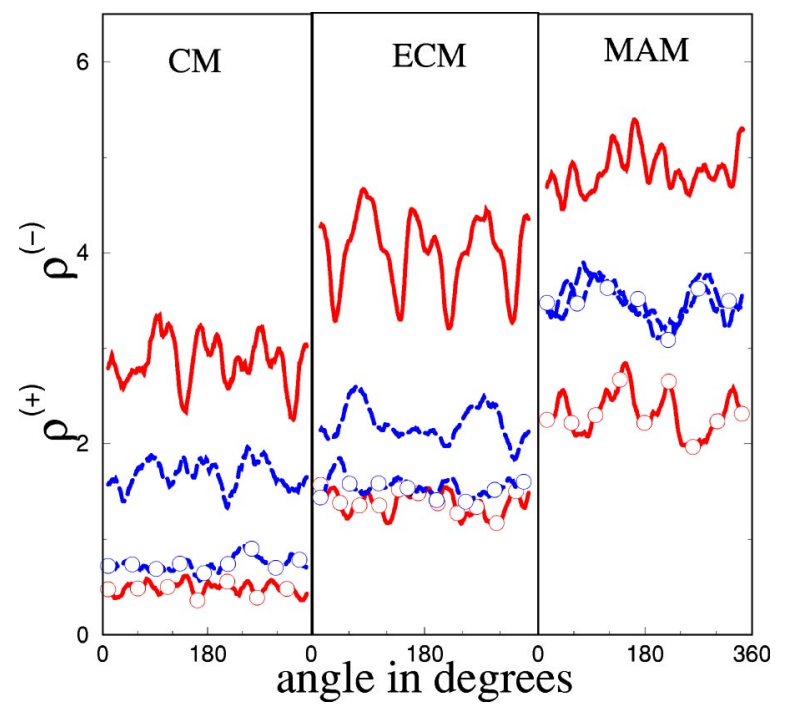

FIG. 11. Panoramic view of ion charge densities in the DNA grooves, monovalent counterions, and divalent salt (set 2) and $C_{s}$ $=1.61 \mathrm{~mol} / \mathrm{l}$. Different DNA models, from left to right: CM, ECM, MAM. Full line: charge distribution in the minor groove; dashed line: charge distribution in the major groove. Lines without or with symbols correspond to cationic $\rho^{(+)}$or anionic $\rho^{(-)}$charge densities. Note that the major-groove neutralization, described by a coincidence of the major-groove cationic (dashed line) and majorgroove anionic (dashed line with symbols) densities, appears only in the MAM.

correlations included [89-91]. In detail, we consider a "physical" overcharging $[35,92]$, when the sign of the total charge of the complex of macroion and small ions, which are localized in a thin shell around the macroion, is opposite to

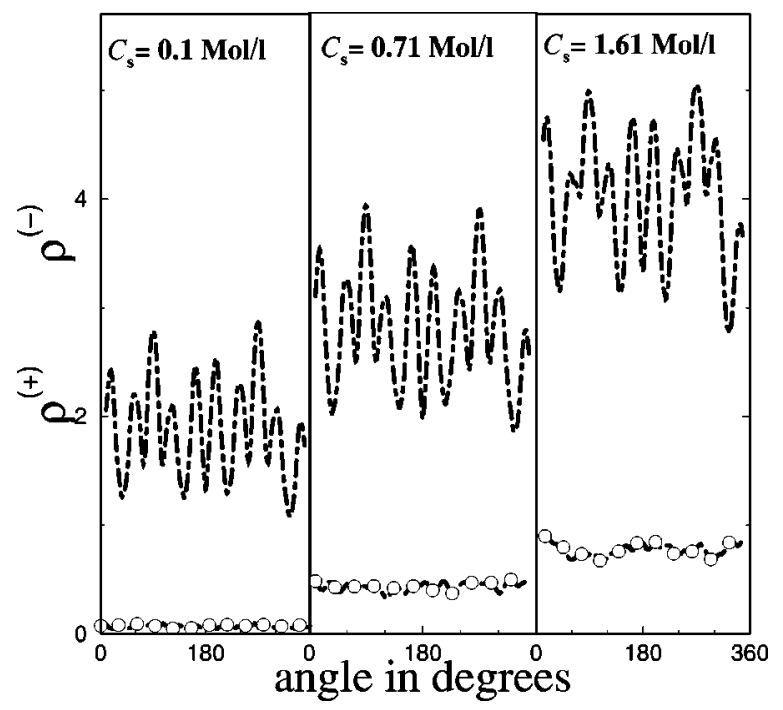

FIG. 12. Panoramic view of ion charge densities on the phosphate strands for the MAM, trivalent counterions, and divalent salt (set 3), and three different salt concentrations; from left to right, $C_{s}=0.1 \mathrm{~mol} / 1,0.71 \mathrm{~mol} / 1,1.61 \mathrm{~mol} / 1$. The total ionic charge on the strand, defined as the difference between the cationic charge $\rho^{(+)}$ (dot-dashed line) and anionic charge $\rho^{(-)}$(dot-dashed line with symbols), increases as more salt is added to solution.

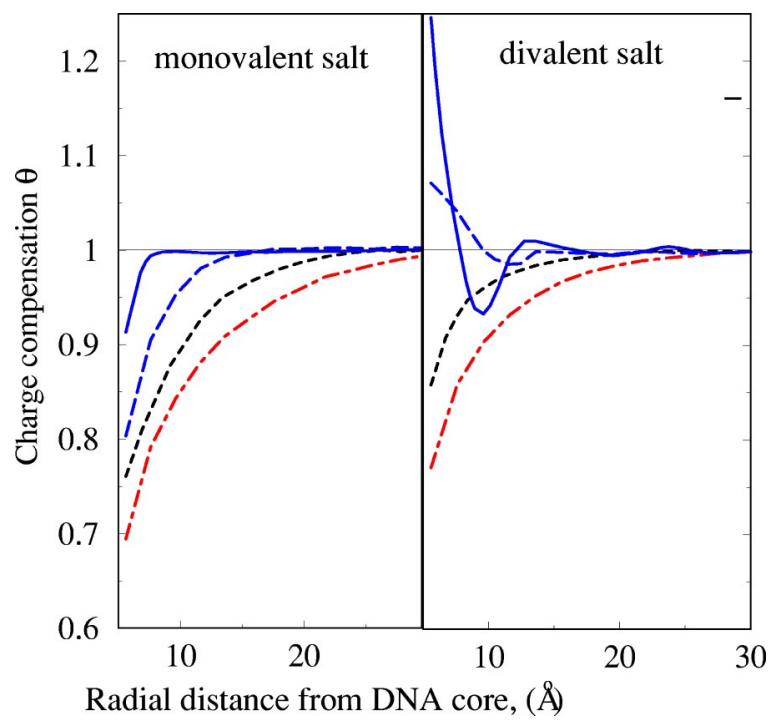

FIG. 13. Charge compensation parameter $\theta$ versus distance from the DNA core surface for the MAM and different salt densities. The salt concentration $C_{s}$ is increased from bottom to top; $0.1 \mathrm{M} / 1$ (dotdashed line), $0.2 \mathrm{M} / \mathrm{l}$ (dashed line), $0.71 \mathrm{M} / 1$ (long dashed line), and $1.61 \mathrm{M} / \mathrm{l}$ (full line). (a) Trivalent counterions and monovalent salt (set 5). (b) Monovalent counterions and divalent salt (set 2).

the sign of the bare macroion charge. Our definition of overcharging differs therefore from the counterion- or so-called Z-ion-induced "structural" overcharging [93-98] which does not account for the adsorbed salt ions and disappears as more salt is added to the solution [99].

During the simulations the charge compensation parameter of DNA phosphate charges, defined as

$$
\theta(r)=\left[q_{c} \rho_{c}(r)+q_{+} \rho_{+}(r)+q_{-} \rho_{-}(r)\right] / N_{p}\left|q_{p}\right|,
$$

was calculated. Here $N_{p}=60$ is the number of phosphate charges in the simulation box. The parameter $\theta(r)$ accounts for the integrated total charge at a distance $r$ away from the DNA surface and has the following physical meaning. For $\theta(r)<1$, the DNA molecule is seen as a negatively charged rod at distance $r$ from its surface. Otherwise, if $\theta(r)>1$, the effective DNA charge at a distance $r$ from its surface is positive. Data for $\theta$ are plotted in Fig. 13 for the MAM and different salt densities. The denser the salt, the stronger the DNA screening. A qualitatively similar picture to Fig. 13 appears for the ECM and CM and different counterion valencies. There is no DNA overcharging in a solution of monovalent salt and multivalent counterions (see the lefthand side of Fig. 13). For a divalent salt,which is shown in the right-hand side of Fig. 13, and low salt densities the compensation parameter is monotonic, resembling the monovalent salt case. However, for dense salt $\theta>1$ in the DNA vicinity. At the highest salt density involved in our simulations, $C_{s}=1.61 \mathrm{~mol} / \mathrm{l}$, there are even several subsequent overcharging layers: within the layer closest to the DNA surface the effective charge is positive, then within the second layer the effective DNA charge is negative, and finally within the third layer the effective charge again becomes positive (see the full line in the right-hand side of Fig. 


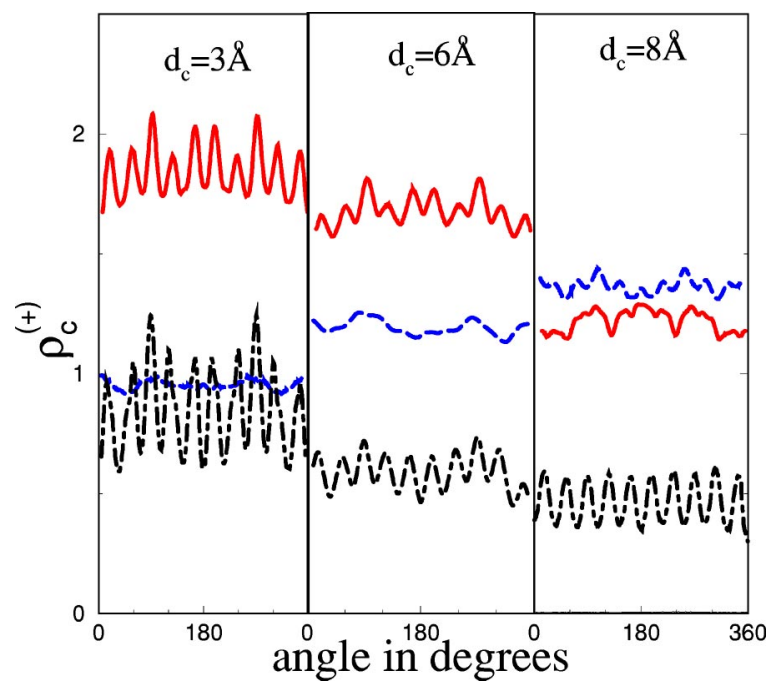

FIG. 14. Panoramic view of cation density near DNA surface for $q_{c}=1, q_{s}=1$ (set 1), $C_{s}=0.1 \mathrm{~mol} / \mathrm{l}$, and the MAM. Different cation diameters, from left to right: $d_{c}=3 \AA, d_{c}=6 \AA, d_{c}=8 \AA$. Full line: cation distribution in the minor groove; dashed line: cation distribution in the major groove; dot-dashed line: cation distribution on the phosphate strands. Note that the cation adsorption in the major groove exceeds the cation adsorption in the minor groove for $d_{c}=8 \AA$ (collate the full and dashed lines in the right side of figure).

13). We mention that similar overcharging pictures are obtained for the divalent and trivalent counterions in solution with a divalent salt. Thus, summarizing the results of Fig. 13, we arrive at the conclusion that it is rather the multivalency of salt ions than the multivalency of counterions which governs DNA overcharging. Our simulations also show that overcharging with a divalent salt in the CM shows up only for multivalent counterions.

\section{E. Varying the ion radius}

The ion size has the meaning of a hydrated ion diameter and is an adjustable parameter of the model that includes effects of the molecular nature of the solvent in an averaged sense. Monovalent counterions are less solvated than divalent cations; the latter condense via their solvent ligands to $\mathrm{H}$ bonds on a DNA surface [100-102]. Since we omit the ion chemisorption and do not account for specific ion effects as exemplified by the Hofmeister effect [103-106], only an electrostatic interaction with the phosphate backbone is taken into account. A small change of the ion size, just in the range of the typical values of hydrated ion diameters, may cause a transition to an attractive DNA-DNA interaction with the spontaneous assembly of DNA system into an ordered phase [41]. Therefore the hydrodynamic ion size affects the ion correlations and the ion condensation on the DNA surface, which are important contributions to the electrostatic potential around the DNA molecule [107,108].

We vary the hydrodynamic radii of solvated ions between 3 and $8 \AA[80,109]$. The simulation results for three different ion diameters, $d_{c}=3,6$, and $8 \AA$ are shown in Fig. 14. The gap between the cationic groove occupations decreases as the ion radius increases. Furthermore, more cations go to the major groove, whereas the minor-groove and the strand ionic occupations shrink. In total, a small amount of cations and anions condense on the DNA surface. Nevertheless, all qualitative findings obtained for ions with diameter $d_{c}=3 \AA$ and outlined in the previous section remain valid for a twice enlarged ion size $d_{c}=6 \AA$. A further increase of the ionic size up to $d_{c}=8 \AA$, however, makes ion intrusion into the minor groove a very rare event. The major-groove cationic charge then exceeds the minor-groove cationic charge (see the right-hand side of Fig. 14). Thus the salt ions "physically" cannot explore the full details of the elaborated DNA model. In other words, the salt ions start to experience the MAM shape as a less elaborated DNA model, like the ECM. Thus the simulation results for the MAM with $d_{c}=8 \AA$ qualitatively resemble the results for the ECM with $d_{c}=6$ or $3 \AA$.

\section{CONCLUSIONS}

In this work we have studied several models of a DNA polyelectrolyte system containing a mixture of mono- and multivalent ions within the framework of a continuum dielectric approach. We have neglected the granular nature of water and concentrated only on the electrostatics of the ion condensation on the DNA surface. Summarizing the results obtained, we have shown that the small ion condensation pattern on the DNA surface strongly depends on the geometry of the DNA model used. While in the simple cylindrical model cations predominantly bind to the phosphate strands, in the more realistic Montoro-Abascal model the minor groove becomes the principal site of cationic binding. Our simulation results also indicate that the anion condensation is less sensitive to the DNA model shape. We have further investigated the occupancy and charging of DNA grooves as functions of increasing cation valency.

We find that the adsorbed ion pattern change with increasing counterion valency is as follows: (i) the cations leave both the major and minor grooves to the phosphate strands, (ii) there are more cations in the minor groove than in the major groove, exhibiting a structuring reminiscent of the spine of solvation, and (iii) the accumulated cationic charges in the major groove are almost independent of the counterion valency.

An increase in salt concentration leads to the following effects. For a monovalent salt, (i) the major groove keeps its total charge at a constant value, and (ii) there is a constant cationic charge asymmetry between the DNA grooves; and for a multivalent salt, (iii) the minor groove keeps its total charge at a constant value, and (iv) the major groove becomes neutralized and a DNA overcharging occurs.

These implications are based on our simulation results, which cover a range of added salt concentrations from 0.1 to $1.61 \mathrm{~mol} / \mathrm{l}$. For monovalent ions this corresponds to a change of $\lambda_{D}$ between 10 and $2 \AA$, a fivefold decrease. The characteristic size (width of the groove along the DNA axis) for the major groove is roughly $16 \AA$ between the edging phosphate surfaces or $20 \AA$ between the phosphate centers. The same parameters for the minor groove are around $10 \AA$ and $14 \AA$, 
respectively. Thus the ratio between $\lambda_{D}$ and the groove size is small, which means that there is effectively no strong interaction between the groove populations. In other words, the groove population is governed mainly by the entropic restriction of ions in the groove, which is different for different DNA models. Unfortunately, due to the time-consuming simulation procedures, equilibrium averages for salt densities beyond $1.61 \mathrm{~mol} / \mathrm{l}$ are difficult to obtain. But, based on the suggestions outlined above, we expect stability of our results for added salt denser than $1.61 \mathrm{~mol} / \mathrm{l}$.

Another issue is the packing fraction of the small ions in the grooves. In our simulations this parameter is small and the ion-ion hard core interaction is not a prevailing factor for the ion distribution in the DNA grooves. However, for larger packing fractions of ions in the grooves, when the grooves are fully packed, our results do not apply.

All these trends represent important information for the implementation of more phenomenological theories for the interaction between two DNA molecules such as the Kornyshev-Leikin theory [37], where the number of condensed ions on the phosphate strands and on the grooves is a key input quantity. As our results indicate, however, one cannot assume constant fractions of adsorbed ions when the salt concentration and/or the counterion valency is varied.

Let us finally discuss some improvements of our model which could be done step by step in a more realistic description of DNA. First, we assume that the persistence length of DNA does not depend from the added salt concentration in order to fit our setup of infinitely stiff single DNA [110121]. The effect of added salt on the DNA stiffness can be taken into account only in simulations with finite DNA fragments. Second, refinement of the present models may account for the specific short-range ion-DNA interactions, or specific "bonding" of ions to the DNA surface, on the basis of effective ion-ion and ion-DNA interactions [36,122]. Such chemisorption is believed to contribute to the force-angle dependence of the DNA-DNA interaction force and could last over distances larger the Debye length, 15-30 $\AA$ $[37,123,124]$. We did a few tentative simulations where some fraction of cations were fixed in the major groove. Prelimi- nary results show no qualitative changes to our conclusions. Of course, many things depend on how many cations are allowed to stick to the major groove, and whether the fraction of these cations must be changed as more salt is added. The same things could be modeled for the minor groove as well. Third, and maybe most important of all, the solvent granularity and the space-dependent dielectric constant were disregarded completely, in a similar way to that adopted in Refs. $[23,41,72,79,80,125-133]$. There are reports that the homogeneous solvent is not as crude as it seems from a first glance $[134,135]$. A more correct approach would be to attribute a significantly lower dielectric constant to the DNA, since the most interesting part of the results comes from ions in the grooves. We are aware that these dielectric effects are difficult to predict without a detailed calculation. A molecular dynamics calculation with a more realistic water model and an atomistic description of DNA would be very demanding. A compromise would be to do "simple" electrostatic calculations on a few key ionic configurations, taking proper account of dielectric effects, to see whether calculated energies and ion occupations change significantly. In computer simulations the dielectric boundaries are usually invoked for spherical and planar colloids. Few works have been devoted to the ion-dielectric cylinder interaction (see Refs. $[82,136,137])$. A modified Coulomb interaction and distancedependent $\epsilon$, in order to mimic the dielectric saturation and discontinuity effects, were addressed in Refs. [80,127,25]. Unfortunately, none of these methods can be directly applied to our model, which consists of a combination of the dielectric cylinder and four strings of dielectric spheres. We leave this as an open issue for future studies.

The other experimentally inspired issue is the mechanics of $B$-DNA which allows the minor groove to open and close to accommodate divalent cations [100]. Taking account of this effect as well as ion chemisorption in the major groove are objectives of future work.

\section{ACKNOWLEDGMENTS}

E.A. thanks R. Podgornik for fruitful discussions of some results of this paper. We thank the DFG for financial support.
[1] W. Saenger, Principles of Nucleic Acid Structure (SpringerVerlag, New York, 1984).

[2] A. Katchalsky, Pure Appl. Chem. 26, 327 (1971).

[3] G.S. Manning, Q. Rev. Biophys. 11, 179 (1978); Acc. Chem. Res. 12, 443 (1979).

[4] P. Mills, C.F. Anderson, and M.T. Record, J. Phys. Chem. 89, 3984 (1985).

[5] P.L. Hansen, R. Podgornik, and V.A. Parsegian, Phys. Rev. E 64, 021907 (2002).

[6] R.A. Marcus, J. Chem. Phys. 23, 1057 (1955).

[7] R. Fuoss, A. Katchalsky, and S. Lifson, Proc. Natl. Acad. Sci. U.S.A. 37, 579 (1951); T. Alfrey, P. Berg, and H. Morawitz, 7, 543 (1951).

[8] C.S. Murthy, R.J. Bacquet, and P.J. Rossky, J. Phys. Chem. 89, 701 (1985).
[9] P. Mills, M.D. Paulsen, C.F. Anderson, and M.T. Record, Jr., Chem. Phys. Lett. 129, 155 (1986).

[10] V. Vlachy and A.D.J. Haymet, J. Chem. Phys. 84, 5874 (1986).

[11] M.D. Paulsen, B. Richey, C.F. Anderson, and M.T. Record, Jr., Chem. Phys. Lett. 139, 448 (1987).

[12] M.D. Paulsen, C.F. Anderson, and M.T. Record, Biopolymers 27, 1249 (1988)

[13] P.J. Lin-Chung and A.K. Rajagopal, Phys. Rev. E 52, 901 (1995).

[14] F. Fogolari, P. Zuccato, G. Esposito, and P. Viglino, Biophys. J. 76, 1 (1999).

[15] J.L. Hecht, B. Honig, Y.K. Shin, and W.L. Hubbell, J. Phys. Chem. 99, 7782 (1995).

[16] S.S. Zakharova, S.U. Egelhaaf, L.B. Bhuiyan, D. Bratko, and 
J.R.C. van der Maarel, J. Chem. Phys. 111, 10706 (1999).

[17] T. Das, D. Bratko, L.B. Bhuiyan, and C.W. Outhwaite, J. Chem. Phys. 99, 410 (1995).

[18] M. Feig and B.M. Pettitt, Biopolymers 48, 199 (1998).

[19] M. Feig and B.M. Pettitt, Biophys. J. 77, 1769 (1999).

[20] M.C. Olmsted, C.F. Anderson, and M.T. Record, Jr., Proc. Natl. Acad. Sci. U.S.A. 86, 7766 (1989).

[21] M.C. Olmsted, J.P. Bond, C.F. Anderson, and M.T. Record, Biophys. J. 68, 634 (1995).

[22] S.A. Allison, J. Chem. Phys. 98, 12091 (1994).

[23] L. Yang, A. Weerasinghe, and B.M. Pettitt, Biophys. J. 69, 1519 (1995).

[24] G. Lamm and G.R. Pack, J. Phys. Chem. B 101, 959 (1997); Biopolymers 34, 227 (1994).

[25] B. Jayaram and D.L. Beveridge, Annu. Rev. Biophys. Biomol. Struct. 25, 367 (1996).

[26] B. Jayapura and K.A. Sharp, Biopolymers 28, 975 (1989).

[27] M.A. Young and D.L. Beveridge, J. Mol. Biol. 281, 675 (1998).

[28] J.N.M. Gurlie and S.C. Harrison, Nature (London) 373, 257 (1995).

[29] J.-P. Hansen and H. Löwen, Annu. Rev. Phys. Chem. 51, 209 (2000).

[30] A.M.J.J. Bonvin, Eur. Biophys. J. 29, 57 (2000).

[31] A.P. Lyubartsev and L. Nordenskiöld, J. Phys. Chem. B 101, 4335 (1997).

[32] N. Korolev, A.P. Lyubartsev, A. Rupprecht, and L. Nordenskiöld, Biophys. J. 77, 2736 (1999).

[33] J.C.G. Montoro and J.L.F. Abascal, J. Chem. Phys. 103, 8273 (1995).

[34] J.C.G. Montoro and J.L.F. Abascal, J. Chem. Phys. 109, 6200 (1998).

[35] J.L.F. Abascal and J.C.G. Montoro, J. Chem. Phys. 114, 4277 (2001).

[36] L.E. Guldbrand, T.R. Forester, and R.M. Lynden-Bell, Mol. Phys. 67, 473 (1989).

[37] A.A. Kornyshev and S. Leikin, Proc. Natl. Acad. Sci. U.S.A. 95, 13579 (1998); Phys. Rev. Lett. 82, 4138 (1999).

[38] R. Netz, Eur. Phys. J. E 5, 557 (2001).

[39] H. Deng, V.A. Bloomfield, J.M. Benevides, and G.J. Thomas, Jr., Nucleic Acids Res. 28, 3379 (2000).

[40] M. Deserno, A. Arnold, and C. Holm, Macromolecules 36, 249 (2003).

[41] A.P. Lyubartsev and L. Nordenskiöld, J. Phys. Chem. 99, 10373 (1995).

[42] H. Deng, V.A. Bloomfield, J.M. Benevides, and G.J. Thomas, Jr., Nucleic Acids Res. 28, 3379 (2000).

[43] H. Deng and V.A. Bloomfield, Biophys. J. 77, 1556 (1999).

[44] D. A. Knoll, M. D. Fried, and V. A. Bloomfield, in DNA and its Drug Complexes, edited by R. H. Sarma and M. H. Sarma (Adenin Press, New York, 1988), p. 123.

[45] D.C. Rau and V.A. Parsegian, Biophys. J. 61, 260 (1992); 61, 246 (1992).

[46] V.A. Bloomfield, Curr. Opin. Struct. Biol. 6, 334 (1996).

[47] N. Korolev, A.P. Lyubartsev, L. Nordenskiöld, and A. Laaksonen, J. Mol. Biol. 38, 907 (2001).

[48] N. Korolev, A.P. Lyubartsev, A. Rupprecht, and L. Nordenskiöld, Biopolymers 58, 268 (2001).

[49] N. Korolev, A.P. Lyubartsev, A. Laaksonen, and L. Norden- skiöld, Biophys. J. 82, 2860 (2002).

[50] J. Sponer, J. Leszczynski, and P. Hobza, Biopolymers 61, 3 (2002).

[51] J. Duguid, V.A. Bloomfield, J. Benevides, and G.J. Thomas, Jr., Biophys. J. 65, 1916 (1993).

[52] S. S. Cohen, A Guide to Polyamines (Oxford University Press, Oxford, 1998).

[53] C.W. Tabor and H. Tabor, Annu. Rev. Biochem. 53, 749 (1984).

[54] H.R. Drew and R.E. Dickerson, J. Mol. Biol. 151, 535 (1981).

[55] B.G. Feuerstein, N. Pattabiraman, and L.J. Marton, Proc. Natl. Acad. Sci. U.S.A. 83, 5948 (1986); Nucleic Acids 18, 1271 (1990).

[56] L.C. Gosule and J.A. Schellman, Nature (London) 259, 333 (1976); J. Mol. Biol. 121, 311 (1978).

[57] R.W. Wilson and V.A. Bloomfield, Biochemistry 18, 2192 (1979); R.W. Wilson, D.C. Rau, and V.A. Bloomfield, Biophys. J. 30, 317 (1980).

[58] M. Le Bret and B. Zimm, Biopolymers 23, 271 (1984); 23, 287 (1984).

[59] E. Allahyarov and H. Löwen, Phys. Rev. E 62, 5542 (2000).

[60] J. Lekner, Physica A 176, 485 (1991); Mol. Simul. 20, 357 (1998).

[61] B. Andreasson, L. Nordenskiöld, W.H. Braunlin, J. Schultz, and P. Stibs, Biochemistry 32, 961 (1993).

[62] W.H. Braunlin, T. Drakenberg, and L. Nordenskiöld, Biopolymers 26, 1047 (1987).

[63] M. Deserno, C. Holm, and S. May, Macromolecules 33, 199 (2000).

[64] S.B. Howerton, C.C. Sines, D. van der Veer, and L.D. Williams, Biochemistry 40, 10023 (2001).

[65] L. McFail-lsom, C.C. Sines, and L.D. Williams, Curr. Opin. Struct. Biol. 9, 298 (1999).

[66] B. Halle and V.P. Denisov, Biopolymers 48, 210 (1998).

[67] V.P. Denisov and B. Halle, Proc. Natl. Acad. Sci. U.S.A. 97, 629 (2000).

[68] M. Soler-Lopez, L. Malinina, J. Liu, T. Huynh-Dinh, and J.A. Subirana, J. Biol. Chem. 274, 23683 (1999).

[69] K.J. McConnell and D.L. Beveridge, J. Mol. Biol. 304, 803 (2000).

[70] B. Schneider, K. Patel, and H.M. Berman, Biophys. J. 75, 2422 (1998).

[71] M.A. Yang, B. Jayaram, and D.L. Beveridge, J. Am. Chem. Soc. 119, 59 (1997).

[72] G.R. Pack, G.A. Garrett, L. Wong, and G. Lamm, Biophys. J. 65, 1363 (1993).

[73] E. Rajasekaran and B. Jayaram, Biopolymers 34, 443 (1994).

[74] M.A. Young, B. Jayaram, and D.L. Beveridge, J. Am. Chem. Soc. 119, 59 (1997).

[75] X. Shui, L. McFail-lsom, G.G. Hu, and L.D. Williams, Biochemistry 37, 8341 (1998).

[76] X. Shui, G. Sines, L. McFail-lsom, D. Van-Derveer, and L.D. Williams, Biochemistry 37, 16877 (1998).

[77] D.B. Tippin and M. Sundaralingam, Biochemistry 36, 536 (1997).

[78] M. Eisenstein and X. Shakked, J. Mol. Biol. 248, 662 (1995).

[79] B. Jayaram, K. Sharp, and B. Honig, Biopolymers 28, 975 (1989). 
[80] J. Conrad, M. Troll, and B.H. Zimm, Biopolymers 27, 1711 (1988).

[81] D. Hochberg, T.W. Kephart, and G. Edwards, Phys. Rev. E 49, 851 (1994); D. Hochberg, G. Edwards, and T.W. Kephart, ibid. 55, 3765 (1997); G. Edwards, D. Hochberg, and T.W. Kephart, ibid. 50, R698 (1994).

[82] D. Stigter, Biopolymers 46, 503 (1998).

[83] G.M. Torrie and J.P. Valleau, J. Chem. Phys. 73, 5807 (1980).

[84] L. Degreve, M. Lozada-Cassou, E. Sancez, and E. GonzalezTovar, J. Chem. Phys. 98, 8905 (1993); L. Degreve and M. Lozada-Cassou, Mol. Phys. 86, 759 (1995).

[85] M. Jonsson and P. Linse, J. Chem. Phys. 115, 3406 (2001).

[86] E.G. Tovar, H. Losada-Cassou, and D. Henderson, J. Chem. Phys. 83, 361 (1985).

[87] H. Greberg and R. Kjellander, J. Chem. Phys. 108, 2940 (1998); J. Ulander, H. Greberg, and R. Kjellander, ibid. 115, 7144 (2001).

[88] R. Kjellander, S. Marcelja, R.M. Pashley, and J.P. Quirk, J. Chem. Phys. 92, 4399 (1990); H. Greberg and R. Kjellander, ibid. 108, 2940 (1998).

[89] S.Y. Park, R.F. Bruinsma, and W.M. Gelbart, Europhys. Lett. 46, 454 (1999).

[90] J.F. Joanny, Eur. Phys. J. B 9, 117 (1999); R.R. Netz and J.F. Joanny, Macromolecules 32, 9026 (1999); 32, 9013 (1999).

[91] F.A.M. Leermakers, J.M.P. van der Oever, and E.B. Zhulina, J. Chem. Phys. 118, 969 (2003).

[92] M. Deserno, F. Jimenez-Angeles, C. Holm, and M. LozadaCassou, J. Phys. Chem. B 105, 10983 (2001); M. QuesadaPerez, E. Gonzalez-Tovar, A. Martin-Molina, M. LozadaCassou, and R. Hidalgo-Alvarez, ChemPhysChem 4, 235 (2003).

[93] R. Messina, C. Holm, and K. Kremer, Phys. Rev. Lett. 85, 872 (2000); Europhys. Lett. 51, 461 (2000).

[94] M. Patra, M. Patriarca, and M. Karttunen, e-print physics/0211006.

[95] T.T. Nguyen, A.Yu. Grosberg, and B.I. Shklovskii, J. Chem. Phys. 113, 1110 (2000); B.I. Shklovskii, Phys. Rev. E 60, 5802 (1999); T.T. Nguyen, A.Yu. Grosberg, and B.I. Shklovskii, Phys. Rev. Lett. 85, 1568 (2000); B.I. Shklovskii, ibid. 82, 3268 (1999).

[96] T.T. Nguyen and B.I. Shklovskii, J. Chem. Phys. 115, 7298 (2001); A.Yu. Grosberg, T.T. Nguyen, and B.I. Shklovskii, Rev. Mod. Phys. 74, 329 (2002).

[97] R.M. Talingting, Y. Ma, C. Simmons, and S.E. Webber, Langmuir 16, 862 (2000).

[98] P.S. Kuhn, Y. Levin, and M.C. Barbosa, Physica A 266, 413 (1999); 274, 8 (1999).

[99] E. Allahyarov and H. Löwen, Phys. Rev. E 63, 041403 (2001).

[100] T.K. Chiu and R.E. Dickerson, J. Mol. Biol. 301, 915 (2000).

[101] H.A. Tajmir-Riahi, M. Naoui, and R. Ahmad, J. Biomol. Struct. Dyn. 11, 83 (1993); I. Fita, J.L. Campos, L.C. Puigjaner, and J.A. Subriana, J. Mol. Biol. 167, 157 (1983).

[102] P.J. Heath and J.M. Schurr, Macromolecules 25, 4149 (1992).

[103] B.W. Ninham and V. Yaminsky, Langmuir 13, 3097 (1997).

[104] A. Tardieu, F. Bonnete, S. Finet, and D. Vivares, Acta Crystallogr., Sect. D: Biol. Crystallogr. 58, 1549 (2002).

[105] R. Piazza and M. Pierno, J. Phys.: Condens. Matter 12, A443 (2000).
[106] P. Benas, L. Legrand, and M. Ries-Kautt, Acta Crystallogr., Sect. D: Biol. Crystallogr. 58, 1582 (2002).

[107] S. Gavryushov and P. Zielenkiewicz, Biophys. J. 75, 2732 (1998).

[108] D. Stigter and K.A. Dill, Biophys. J. 71, 2067 (1996).

[109] B. E. Conway, Ionic Hydration in Chemistry and Biophysics (Elsevier, Amsterdam, 1981), p. 73.

[110] L.D. Williams and L.J. Maher III, Annu. Rev. Biophys. Biomol. Struct. 29, 497 (2000).

[111] Y. Lu, B. Weers, and N.C. Stellwagen, Biopolymers 61, 261 (2002).

[112] G. Ariel and D. Andelman, Phys. Rev. E 67, 011805 (2003); Europhys. Lett. 61, 67 (2003).

[113] J.G. Duguid and V.A. Bloomfield, Biophys. J. 69, 2642 (1995); J.G. Duguid et al. ibid. 69, 2623 (1995).

[114] P.E. Sottas, E. Larquet, A. Stasiak, and J. Dubochet, Biophys. J. 77, 1858 (1999).

[115] M.O. Khan and B. Jönsson, Biopolymers 49, 121 (1999).

[116] C.G. Baumann, S.B. Smith, V.A. Bloomfield, and C. Bustamante, Proc. Natl. Acad. Sci. U.S.A. 94, 6185 (1997).

[117] K.K. Kunze and R.R. Netz, Phys. Rev. E 66, 011918 (2002).

[118] H. Liu, J. Gapinski, L. Skibinska, A. Patkowski, and R. Pecora, J. Chem. Phys. 113, 6001 (2000).

[119] J. Widom and R.L. Baldwin, J. Mol. Biol. 144, 431 (1980).

[120] V.A. Bloomfield, Biopolymers 44, 269 (1997).

[121] R. Golestanian, M. Kardar, and T.B. Liverpool, Phys. Rev. Lett. 82, 4456 (1999).

[122] B.M. Pettitt and P.J. Rossky, J. Chem. Phys. 84, 5836 (1986).

[123] H.M. Harreis, A.A. Kornyshev, C.N. Likos, H. Löwen, and G. Sutmann, Phys. Rev. Lett. 89, 018303 (2002); Virt. J. Biol. Phys. Res. 3, 12 (2002).

[124] A.G. Cherstvy, A.A. Kornyshev, and S. Leikin, J. Phys. Chem. B 106, 13362 (2002).

[125] P.G. Kusalik and G.N. Patey, J. Chem. Phys. 88, 7715 (1988).

[126] M.K. Gilson, A. Rashin, R. Fine, and B. Honig, J. Mol. Biol. 183, 503 (1985).

[127] B. Jayaram, S. Swaminathan, D.L. Beveridge, K. Sharp, and B. Honig, Macromolecules 23, 3156 (1990); B. Jayaram, A. Das, and N. Aneja, J. Mol. Struct. 361, 249 (1996).

[128] M.A. Young, B. Jayaram, and D.L. Beveridge, J. Phys. Chem. B 102, 7666 (1998).

[129] J. Mazur and R.L. Jernigan, Biopolymers 31, 1615 (1991).

[130] D. Petsev, B.R. Thomas, S.T. Yau, and P.G. Vekilov, Biophys. J. 78, 2060 (2000).

[131] A.V. Lukashin, D.B. Beglov, and M.D. Frank-Kamenetskii, J. Biomol. Struct. Dyn. 9, 517 (1991).

[132] J.R.C. van der Maarel, Biophys. J. 76, 2673 (1999).

[133] The dielectric discontinuity effect for spherical geometry and image charges was recently analyzed in R. Messina, J. Chem. Phys. 117, 1 (2002). See also P. Linse, J. Phys. Chem. 90, 6821 (1986); G.M. Torrie, J.P. Valleau, and G.N. Patey, J. Chem. Phys. 76, 4615 (1982).

[134] See L.R. Pratt, G. Hummer, and A.E. Garsia, Biophys. Chem. 51, 147 (1994); E. Guardia, R. Rey, and J.A. Padro, J. Chem. Phys. 95, 2823 (1991).

[135] A.P. Lyubartsev and A. Laaksonen, Phys. Rev. E 52, 3730 (1995).

[136] M. Troll, D. Roitman, J. Conrad, and B.H. Zimm, Macromolecules 19, 1186 (1986).

[137] J. Skolnik and M. Fixman, Macromolecules 11, 867 (1978). 